\title{
A GEOMETRIC CONSTRUCTION OF TANGO BUNDLE ON $\boldsymbol{P}^{5}$
}

\author{
DANIELE FAENZI
}

\begin{abstract}
The Tango bundle $T$ over $\boldsymbol{P}^{5}$ is proved to be the pull-back of the twisted Cayley bundle $C(1)$ via a map $f: \boldsymbol{P}^{5} \rightarrow Q_{5}$ existing only in characteristic 2 . The Frobenius morphism $\varphi$ factorizes via such $f$.
\end{abstract}

\section{Introduction}

The well-known Hartshorne conjecture states, in particular, that there are no indecomposable rank-2 vector bundles on $\boldsymbol{P}^{n}$, when $n$ is greater than 5 . However, one of the few rank-2 bundles on $\boldsymbol{P}^{5}$ up to twist and pull-back by finite morphisms is the Tango bundle $T$ first given in [Tan76]. Later Horrocks in [Hor78] and Decker Manolache and Schreyer in [DMS92] discovered that it can be obtained starting from Horrocks rank-3 bundle: anyway it only exists in characteristic 2 .

Here we prove that $T$ is the pull-back of the twisted Cayley bundle $C(1)$ (defined over any field) via a map $f: \boldsymbol{P}^{5} \rightarrow Q_{5}$ existing only in characteristic 2 . In section (2) we introduce the involved bundles and state the theorems, while in section (3) we give proofs and some more remarks. Also, we give an explanation of why one cannot hope to extend this construction to other characteristics. We make use of Macaulay2 computer algebra package, see [GS].

Aknowledgements. I would like to thank Wolfram Decker and Edoardo Ballico for useful suggestions and the referee for pointing out several improvements. Also I am indebted with Giorgio Ottaviani, who posed to me this problem.

So let $Q_{5}$ be the smooth 5-dimensional quadric hypersurface over an algebraically closed field $k$.

$$
Q_{5}=\left\{z_{0}^{2}+z_{1} z_{2}+z_{3} z_{4}+z_{5} z_{6}=0\right\} \subset \boldsymbol{P}^{6}
$$

We denote by $\xi$ (respectively $\eta, \zeta$ ) the generator of $A^{1}\left(\boldsymbol{P}^{5}\right)$ (respectively of $\left.A^{1}\left(Q_{5}\right), A^{3}\left(Q_{5}\right)\right)$, so that

2000 Mathematics Subject Classification: Primary 14F05; Secondary 14-04, 14J60.

The author was partially supported by Italian MIUR funds and the EAGER net, contract HPRN-CT-2000-00099.

Received November 28, 2001; revised June 10, 2003. 


$$
A\left(\boldsymbol{P}^{5}\right)=\boldsymbol{Z}[\xi] /\left(\xi^{6}\right) \quad A\left(Q_{5}\right)=\boldsymbol{Z}[\eta, \zeta] /\left(\eta^{3}-2 \zeta, \eta^{6}\right)
$$

On the coordinate ring $R\left(\boldsymbol{P}^{5}\right)$ we use variables $x_{i}$ 's while on $R\left(Q_{5}\right)$ we use $z_{j}$ 's.

\section{The bundles on $\boldsymbol{P}^{5}$ and on $Q_{5}$}

Let $k$ be an algebraically closed field. On $Q_{5}=\mathrm{G}_{2} / P\left(\alpha_{1}\right)$ we have the Cayley bundle $C$, associated to the standard representation of the semisimple part of the parabolic group $P\left(\alpha_{1}\right)$, where $\alpha_{1}$ is the shortest root in the Lie algebra of the exceptional Lie group $\mathrm{G}_{2} . \quad C$ is irreducible $\mathrm{G}_{2}$-homogeneous with maximal weight $\lambda_{2}-2 \lambda_{1} . \quad C(2)$ (weight $\lambda_{2}$ ) is globally generated and $\mathrm{h}^{0}(C(2))=14$.

Recall the definition of the spinor 4-bundle $S$ over $Q_{5}$. Since $Q_{5}=$ $\operatorname{Spin}(7) / P\left(\beta_{1}\right)$, where $\beta_{1}$ is the shortest root of $\operatorname{Spin}(7)$, we define $S$ as the bundle associated to the spin representation of the semisimple part $\operatorname{ss}\left(P\left(\beta_{1}\right)\right)=$ Spin(5). $\quad S^{\vee}=S(1)$ is globally generated.

$C$ is related to $S$ in the following way. First one computes $c_{4}\left(S^{\vee}\right)=0$ so there exists a rank-3 bundle $G$ given by

$$
0 \rightarrow \mathcal{O} \stackrel{a}{\rightarrow} S^{\vee} \rightarrow G \rightarrow 0
$$

It turns out that $c_{3}\left(G^{\vee}(1)\right)=0$. One can prove that $G^{\vee}(1)$ has a unique section $b$ and that the quotient by such $b$ is isomorphic to $C(1)$ i.e. $C$ is the cohomology of the monad:

$$
\mathcal{O}(-1) \stackrel{b(-1)}{\longrightarrow} S \stackrel{a^{t}}{\longrightarrow} \mathcal{O}
$$

$C$ has rank 2 and Chern classes $(-1,1)$. The only non-vanishing intermediate cohomology groups are $\mathrm{H}^{1}(C)=\mathrm{H}^{4}(C(-4))=k$. All this is done in [Ott90] and follows easily from [Jan87, Proposition 5.4] in any characteristic.

THEOREM 1. The stable rank-3 bundle $G$ defined on $Q_{5}$ by the sequence (1) is isomorphic to $\operatorname{ker}(\tilde{L})^{\vee}(-2)$ where $\tilde{L}$ is the morphism $\mathcal{O}_{Q_{5}}(-2)^{6} \rightarrow \mathcal{O}_{Q_{5}}^{12}$ defined by the matrix $A$

$$
\left(\begin{array}{cccccc}
z_{0}^{2} & 0 & 0 & z_{1}^{2} & z_{1} z_{3}+z_{0} z_{6} & -z_{0} z_{4}+z_{1} z_{5} \\
0 & z_{0}^{2} & 0 & z_{1} z_{3}-z_{0} z_{6} & z_{3}^{2} & z_{0} z_{2}+z_{3} z_{5} \\
0 & 0 & z_{0}^{2} & z_{0} z_{4}+z_{1} z_{5} & -z_{0} z_{2}+z_{3} z_{5} & z_{5}^{2} \\
0 & z_{0} z_{2}-z_{3} z_{5} & z_{3}^{2} & -z_{0} z_{3}-z_{2} z_{6} & 0 & z_{2}^{2} \\
z_{5}^{2} & 0 & z_{0} z_{4}-z_{1} z_{5} & z_{4}^{2} & -z_{2} z_{4}-z_{0} z_{5} & 0 \\
-z_{3}^{2} & z_{1} z_{3}+z_{0} z_{6} & 0 & -z_{6}^{2} & 0 & -z_{0} z_{3}+z_{2} z_{6} \\
0 & z_{5}^{2} & -z_{0} z_{2}-z_{3} z_{5} & -z_{2} z_{4}+z_{0} z_{5} & z_{2}^{2} & 0 \\
z_{1} z_{3}-z_{0} z_{6} & -z_{1}^{2} & 0 & 0 & -z_{6}^{2} & z_{0} z_{1}+z_{4} z_{6} \\
z_{0} z_{4}+z_{1} z_{5} & 0 & -z_{1}^{2} & 0 & -z_{0} z_{1}+z_{4} z_{6} & -z_{4}^{2} \\
-z_{2}^{2} & -z_{2} z_{4}-z_{0} z_{5} & z_{0} z_{3}-z_{2} z_{6} & z_{0}^{2} & 0 & 0 \\
z_{2} z_{4}-z_{0} z_{5} & z_{4}^{2} & z_{0} z_{1}+z_{4} z_{6} & 0 & z_{0}^{2} & 0 \\
-z_{0} z_{3}-z_{2} z_{6} & z_{0} z_{1}-z_{4} z_{6} & -z_{6}^{2} & 0 & 0 & -z_{0}^{2}
\end{array}\right)
$$


Now we show how to induce a bundle over $\boldsymbol{P}^{5}$ in case $\operatorname{char}(k)=2$.

THEOREM 2. Let $\operatorname{char}(k)=2$. For any odd $n$ there exists a non-constant morphism $f: \boldsymbol{P}^{n} \rightarrow Q_{n}$. For $n=5$ the pull-back $T=f^{*}(C(1))$ is a stable rank-2 bundle with $c_{1}(t)=2, c_{2}(T)=4$ and

$$
\chi(T(t))=\frac{1}{60} t^{5}+\frac{1}{3} t^{4}+\frac{25}{12} t^{3}+\frac{11}{3} t^{2}-\frac{51}{10} t-14
$$

T coincides with the bundle defined by Tango in [Tan76].

\section{Proof of the theorems}

The presentation matrix $A$ in theorem 1 is obtained by stacking 9 rows to Tango's $3 \times 6$ matrix i.e. the first three rows of $A$. The 9 extra rows are obtained by adding convenient expressions, all algebraically dependent over the quotient ring $R\left(Q_{5}\right)=k\left[z_{0}, \ldots, z_{6}\right] /\left(z_{0}^{2}+z_{1} z_{2}+z_{3} z_{4}+z_{5} z_{6}\right)$, in order to get $A$ having everywhere rank 3 over $R\left(Q_{5}\right)$. For example the bottom row $A_{11}$ of $A$ is the following expression of the first three rows

$$
A_{11}=\frac{1}{z_{0}}\left(z_{3} A_{0}-z_{1} A_{1}\right)-\frac{1}{z_{0}^{2}}\left(z_{2} z_{6} A_{0}+z_{4} z_{6} A_{1}+z_{6}^{2} A_{2}\right)
$$

Then the rank of $A$ over any point of $Q_{5}$ is at most 3 and actually it is not hard to show (by exhibiting a sufficient number of minors) that such rank is constantly 3. Thus $A$ defines a rank-3 bundle. Denote by $L$ the morphism of $R\left(Q_{5}\right)$ modules defined by $A, \tilde{L}$ its sheafification and $G^{\prime \vee}=\operatorname{ker} \tilde{L}(2)$.

The rank of $A$ can also be computed by Macaulay2. One can also compute global sections and actually all the cohomology. It turns out that $\mathrm{H}^{0}\left(G^{\prime}\right)=$ $\mathrm{H}^{0}\left(\bigwedge^{2} G^{\prime}\right)=0$ and

$$
\begin{aligned}
\mathrm{h}^{i}\left(Q_{5}, \operatorname{ker} \tilde{L}(t)\right) & =0 \quad \forall t, 0<i<5, \text { except: } \\
\mathrm{h}^{1}\left(Q_{5}, \operatorname{ker} \tilde{L}(2)\right) & =1
\end{aligned}
$$

Let us now compute its Chern classes i.e. we prove the analogue for $Q_{5}$ of [Tan76, lemma 1].

Lemma 3. Let $\rho: Q_{n} \rightarrow \boldsymbol{G}\left(\boldsymbol{P}^{k}, \boldsymbol{P}^{n}\right)$ be a non-constant morphism, with $n$ odd and $k$ even, and let $\mathscr{E}$ be the pull-back on $Q_{n}$ of the dual universal sub-bundle $U^{\vee}$ on $\boldsymbol{G}\left(\boldsymbol{P}^{k}, \boldsymbol{P}^{n}\right)$. Then $k=(n-1) / 2$, and:

$$
c_{i}(\mathscr{E})=2 a^{i}
$$

for some positive integer $a$.

Proof. The proof is almost identical to the case of $\boldsymbol{P}^{n}$, the only difference being that we have to work in $H^{*}\left(Q_{n}\right)$, where $\eta^{(n+1) / 2}=2 \zeta$. 
We can suppose $k$ even and $k \leq(n-1) / 2$ because $\boldsymbol{G}\left(\boldsymbol{P}^{k}, \boldsymbol{P}^{n}\right) \cong$ $\boldsymbol{G}\left(\boldsymbol{P}^{n-k-1}, \boldsymbol{P}^{n}\right)$ and we put $a_{i}=c_{i}\left(\rho^{*}(\mathscr{E})\right), b_{i}=c_{i}\left(\rho^{*}(Q)\right)$ where $Q$ is the universal quotient bundle. We have, in the ring $A\left(Q_{5}\right)[t]$ the relation on Chern polynomials

$$
c_{\mathscr{O} \vee}(t) \cdot c_{\rho^{*}(Q)}(t)=1
$$

Now we can think of the coefficients in (3) as integers times some $\eta^{r}$, taking care to replace $\zeta$ by $\frac{1}{2} \eta^{(n+1) / 2}$, that is, replacing $a_{i}$ (and $b_{i}$ ) by $a_{i}^{\prime}=(1 / 2) a_{i}$ (by $\left.b_{i}^{\prime}=(1 / 2) b_{i}\right)$ whenever $i \geq(n+1) / 2$.

Then one proceeds exactly as in [Tan76, lemma 1] and [Tan74, lemma 3.3], and finds:

$$
\begin{aligned}
k & =\frac{n-1}{2} \\
a_{i}^{\prime} & =2 a^{i} \text { for } i=1, \ldots, \frac{n-1}{2}=k \\
a_{k+1}^{\prime} & =a^{k+1}
\end{aligned}
$$

and so $a_{i}=2 a^{i}$, for all $i$ 's, as only for $i=k+1$ we have to substitute $a_{k+1}=2 a_{k+1}^{\prime}$.

Here $\rho$ is given by the matrix $A$, associating to $x \in Q_{5}$ the 3 -space $A(x)$. We have $\rho^{*}(U)=G^{\mathrm{v}}$. It is easy to prove that ker $\tilde{L}(2)$ contains the line bundle $\mathcal{O}(-1)$, under the linear map given by the vector $v$

$$
v=\left(z_{1}, z_{3}, z_{5}, z_{2}, z_{4}, z_{6}\right)^{t} \quad A \cdot v=0
$$

Then the factor $1-\eta$ must divide the total Chern class. By the above lemma this implies $a=1$. Then the Chern classes $c_{1}, c_{2}, c_{3}$ of $G^{\prime \vee}$ are 2,2,2. We know that $G^{\prime}$ is stable since we have computed global sections. We are now in position to use [Ott88, theorem 3.2] to conclude that

$$
G \cong G^{\prime}
$$

This finishes the proof of theorem (1). Notice that by $\operatorname{Ext}^{1}\left(G^{\prime}, \mathcal{O}\right)=k$ we get a unique extension of the form

$$
0 \rightarrow \mathcal{O} \rightarrow W \rightarrow G^{\prime} \rightarrow 0
$$

where $W$ is a 4-bundle whose intermediate cohomology is forced to be zero. Then by [BEH87] or [BGS87] and Euler characteristic we get $W=S^{\vee}$. This allows to recover the extension (1).

Let us now turn to theorem (2). The map $f$ is defined for any odd $n$ as

$$
f\left(x_{0}: \cdots: x_{n}\right)=\left(x_{0} x_{1}+\cdots+x_{n-1} x_{n}: x_{0}^{2}: \cdots: x_{n}^{2}\right)
$$

it is a finite morphism of degree $2^{n-1}$ and factors the Frobenius morphism $\varphi$ as in the diagram 


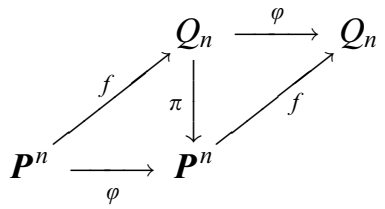

where $\pi$ the projection from $(1: 0: \cdots: 0)$. Then we have $f^{*}\left(\eta^{j}\right)=2^{j} \xi^{j}$ and $f^{*}(\zeta)=2^{(n+1) / 2-1} \xi^{(n+1) / 2}$. In the case $n=5$ we are allowed to define $T=$ $f^{*}(C(1))$. We get $c_{1}(T)=2, c_{2}(T)=4$ and the Hilbert polynomial follows by Hirzebruch-Riemann-Roch.

Recall Tango's definition of the bundle $T^{\prime}$ as quotient of $\operatorname{ker} \tilde{L}(2)$ by the line bundle $\mathcal{O}(-1)$. Then the last claim in theorem (2) follows, since we have shown that $G=G^{\prime}$ and that $G^{\vee}(1)$ has a unique section. Equivalently one can use [Ott90, main theorem] to show that the quotient of $G^{\prime}(1)$ by $\mathcal{O}$ is isomorphic to $C(1)$.

Remark 4. Let $\operatorname{char}(k)=p$. Then, as Edoardo Ballico pointed out to us, we may relate this framework to a description given by Ekedahl in [Eke87, proposition 2.5] of finite degree $p$ morphisms $\psi$ (with $Y$ smooth) that factor the Frobenius $\varphi$

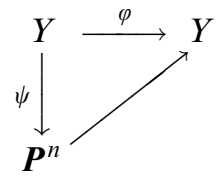

Ekedahl shows that in such a situation $Y$ is $Q_{n}, n$ is odd, $\psi$ is the projection from a point external to $Q_{n}$ and the characteristic is 2 . That is, precisely our setup.

Remark 5. We can provide the resolution of $C(2)$ over $Q_{5}$ with the aid of Macaualy2. We get a resolution of the form

$$
R^{\bullet} \stackrel{\delta}{\rightarrow} \mathcal{O}_{Q_{5}}(-3)^{55} \rightarrow \mathcal{O}_{Q_{5}}(-2)^{49} \rightarrow \mathcal{O}_{Q_{5}}(-1)^{34} \rightarrow \mathcal{O}_{Q_{5}}^{14} \rightarrow C(2) \rightarrow 0
$$

where $R^{\bullet}$ a 2-periodic complex, cfr. [Eis80], of the form $\cdots \rightarrow \mathcal{O}_{Q_{5}}(i)^{56} \rightarrow$ $\mathcal{O}_{Q_{5}}(i+1)^{56} \rightarrow \cdots . \quad \operatorname{Im}(\delta)$ is a rank-28 vector bundle and again it must have no intermediate cohomology. Then Euler characteristic shows that it must be $S(-3)^{\oplus 7}$, i.e. the resolution actually reads:

$$
\begin{aligned}
0 \rightarrow S(-3)^{\oplus 7} & \rightarrow \mathcal{O}_{Q_{5}}(-3)^{55} \rightarrow \mathcal{O}_{Q_{5}}(-2)^{49} \\
& \rightarrow \mathcal{O}_{Q_{5}}(-1)^{34} \rightarrow \mathcal{O}_{Q_{5}}^{14} \rightarrow C(2) \rightarrow 0
\end{aligned}
$$

Although we will not consider this here, we remark that the cohomology of $T$ can be computed in terms of the cohomology of $C$ and $C \otimes S$, making use of the projection formula for $f$ and carefully using an analogue of Borel-Bott-Weil theorem in positive characteristic. 


\section{REFERENCES}

[BEH87] Ragnar-Olaf Buchweitz, David Eisenbud, and Jürgen Herzog, Cohen-Macaulay modules on quadrics, Singularities, representation of algebras, and vector bundles (Lambrecht, 1985), Lecture Notes in Math., vol. 1273, Springer, Berlin, 1987, pp. 58-116. MR 89g: 13005

[BGS87] Ragnar-Olaf Buchweitz, Gert-Martin Greuel, and Frank-Olaf Schreyer, CohenMacaulay modules on hypersurface singularities. II, Invent. Math. 88 (1987), no. 1, 165182. MR 88d: 14005

[DMS92] Wolfram Decker, Nicolae Manolache, and Frank-Olaf Schreyer, Geometry of the Horrocks bundle on $\boldsymbol{P}^{5}$, Complex projective geometry (Trieste, 1989/Bergen, 1989), London Math. Soc. Lecture Note Ser., vol. 179, Cambridge Univ. Press, Cambridge, 1992, pp. 128-148. MR 94h:14043

[Eis80] David EISENBud, Homological algebra on a complete intersection, with an application to group representations, Trans. Amer. Math. Soc. 260 (1980), no. 1, 35-64. MR 82d:13013

[Eke87] ToRsten Ekedahl, Foliations and inseparable morphisms, Algebraic geometry, Bowdoin, 1985 (Brunswick, Maine, 1985), Proc. Sympos. Pure Math., vol. 46, Amer. Math. Soc., Providence, RI, 1987, pp. 139-149. MR 89d:14049

[GS] Daniel R. Grayson and Michael E. Stillman, Macaulay 2, a software system for research in algebraic geometry, Available at http://www.math.uiuc.edu/Macaulay2/.

[Hor78] Geoffrey Horrocks, Examples of rank three vector bundles on five-dimensional projective space, J. London Math. Soc. (2) 18 (1978), no. 1, 15-27. MR 80d:14011

[Jan87] Jens CARSTEn JANTZen, Representations of algebraic groups, Pure and Applied Mathematics, vol. 131, Academic Press Inc., Boston, MA, 1987. MR 89c:20001

[Ott88] Giorgio Ottaviani, Spinor bundles on quadrics, Trans. Amer. Math. Soc. 307 (1988), no. 1, 301-316. MR 89h:14012

[Ott90] —, On Cayley bundles on the five-dimensional quadric, Boll. Un. Mat. Ital. A (7) 4 (1990), no. 1, 87-100. MR 91c:14055

[Tan74] Hiroshi Tango, On $(n-1)$-dimensional projective spaces contained in the Grassmann variety $\operatorname{Gr}(n, 1)$, J. Math. Kyoto Univ. 14 (1974), 415-460. MR 51 \#8132

[Tan76] - On morphisms from projective space $\boldsymbol{P}^{n}$ to the Grassmann variety $\operatorname{Gr}(n, d), \quad \mathrm{J}$. Math. Kyoto Univ. 16 (1976), no. 1, 201-207. MR 53 \#5614

Dipartimento di Matematica

"U. Dini", Università di FirenZE

Viale Morgagni 67/a, I-50134, Florence, Italy

e-mail: faenzi@math.unifi.it

URL: http://www.math.unifi.it/ faenzi/ 